Pacific Journal of Mathematics

ON SOME COVERING AND INTERSECTION PROPERTIES IN 


\title{
ON SOME COVERING AND INTERSECTION PROPERTIES IN MINKOWSKI SPACES
}

\author{
B. GRÜNBAUM
}

1. Introduction Let $X$ denote a Minkowski space (i.e. a finite dimensional normed linear space over the reals) and let $S=\{x \in X ;\|x\| \leq 1\}$ denote the unit cell of $X$. In this note we shall be concerned with two numbers, $E_{X}$ and $J_{X}$, determined by the geometric properties of $X$.

DeFINITION 1. The expansion constant $E_{X}$ of $X$ is the greatest lower bound of real numbers $\mu \geq 0$ which possess the following property:

Given any family $\left\{x_{i}+\alpha_{i} S ; i \in I\right\}$ of mutually intersecting cells (in other words, given any family $\left\{x_{i}\right\}$ of points and any family $\left\{\alpha_{i}\right\}$ of non-negative numbers such that $\left\|x_{i}-x_{j}\right\| \leq \alpha_{i}-\alpha_{j}$ for all $\left.i, j \in I\right)$; then

$$
\bigcap_{i \in I}\left(x_{i}+\mu \alpha_{i} S\right) \neq \phi .
$$

DeFinition 2. Jung's constant $J_{X}$ of $X$ is the greatest lower bound of real numbers $\mu$ which possess the following property:

Given any family $\left\{x_{i}+S ; i \in I\right\}$ of mutually intersecting cells (i.e. given any family $\left\{x_{i}\right\}$ such that $\left\|x_{i}-x_{j}\right\| \leq 2$ for all $\left.i, j \in I\right\}$; then

$$
\bigcap_{i \in I}\left(x_{i}+\mu S\right) \neq \phi
$$

We note the following immediate consequences of the above definitions :

(i) By Helly's theorem on intersections of convex sets, the index set $I$ may be assumed to consist of not more than $n+1$ elements, where $n$ is the dimension of $X$.

(ii) Standard compactness arguments show that $E_{X}$ and $J_{X}$ are not only the greatest lower bounds, but even the minima of the numbers $\mu$ defining them.

(iii) $1 \leq J_{X} \leq E_{X} \leq 2$.

(iv) $J_{X}$ may equivalently be defined as the smallest number such that a cell of that diameter may cover, after a suitable translation, any set of diameter $\leq 1$.

Received November 12, 1958. The results of this paper form part of Chapter 5 of the author's Ph. D. thesis, "On some properties on Minkowski space" (in Hebrew), prepared under the guidance of Professor A. Dvoretzky at The Hebrew University in Jerusalem. The author wishes to express his sincere gratitude to Professor Dvoretzky for his helpful suggestions and criticism. The results have also been incorporated in a report on "Extensions, Retractions, and Projection ", prepared in part under Contract AF 61 (052)-04. 
The definitions of $E_{X}$ and $J_{X}$ apply obviously to more general spaces. We will show elsewhere [4] that $E_{X}$ plays an important role in the problem of extensions of transformations. In this context Nachbin [9] and Aronszajn-Panitchpakdi [1] have studied concepts closely related to the property $E_{X}=1$.

Szökefalvi-Nagy [12] and Hanner [6] proved that Minkowski spaces whose Jung constant equals unity are precisely those having a parallelohedron as unit cell; the corresponding result for the expansion constant had been established by Nachbin [9].

For the Euclidean space $E^{n}$ the value $J_{E^{n}}=[2 n /(n+1)]^{\frac{1}{2}}$ was determined by Jung [7]. Bohnenblust [2] proved that $J_{X} \leq 2 n /(n+1)$ if $X$ is an $n$-dimensional Minkowski space. Leichtweiss [8] gave a simpler proof of Bohnenblust's result and characterized those $n$-dimensional Minkowski spaces $X$ for which $J_{X}=2 n /(n+1)$.

The main object of the present note is the determination of the exact upper bound of $E_{X}$ for $n$-dimensional spaces $X$ and the characterization of those spaces for which the bound is attained.

2. Bound for the expansion constants. We show first of all that Bohnenblust's bounds for Jung's constants apply also to expansion constants.

TheOREM 1. If $X$ is an n-dimensional Minkowski space then $E_{X} \leq 2 n /(n+1)$.

Proof. Let $\mathscr{S}=\left\{x_{i}+\alpha_{i} S ; i \in I\right\}$ be any family of mutually intersecting cells in $X$. We shall show that

$$
\bigcap_{i \in I}\left(x_{i}+\frac{2 n}{n+1} \alpha_{i} S\right) \neq \phi .
$$

As remarked above in (i), it is sufficient to prove (1) in case $I$ has $n+1$ elements; we shall assume $I=\{0,1, \cdots, n\}$. We shall also assume that $\alpha_{i}>0$ for each $i \in I$; indeed if $\alpha_{i}=0$ then $x_{i}$ belongs to all members of $\mathscr{S}$ and (1) obviously holds.

Let us denote

$$
\alpha=\sum_{i=0}^{n} \alpha_{i} .
$$

It is convenient to deal separately with two possible cases:

$$
\text { (i) } \alpha \geq(n-1) \alpha_{k} \text { for all } k \in I
$$

(ii) there exists a $k \in I$ such that $\alpha<(n-1) \alpha_{k}$.

In both cases we shall establish (1) by exhibiting a point belonging to 


$$
\bigcap_{i=0}^{n}\left(x_{i}+\mu \alpha_{i} S\right)
$$

for a suitable $\mu \leq 2 n /(n+1)$.

We consider first the case (i) and we introduce the following notations:

$$
\begin{gathered}
\alpha_{i}^{\prime}=1 / \alpha_{i} \quad \text { for } i \in I \\
\alpha^{\prime}=\sum_{i=0}^{n} \alpha_{i}^{\prime} \\
\sigma=\sum_{0 \leq i \leq j \leq n}\left(\alpha_{i} \alpha_{j}^{\prime}+\alpha_{i}^{\prime} \alpha_{j}\right)=\alpha \alpha^{\prime}-(n+1) .
\end{gathered}
$$

Since $\alpha_{i} \alpha_{j}^{\prime}+\alpha_{i}^{\prime} \alpha_{j} \geq 2$, and $\alpha_{i} \alpha_{j}^{\prime}+\alpha_{i}^{\prime} \alpha_{j}=2$ if and only if $\alpha_{i}=\alpha_{j}$, we have

$$
\sigma=n(n+1)+\delta \text { with } \delta \geq 0
$$

and

( 8 )

$$
\delta=0 \text { if and only if } \alpha_{0}=\alpha_{1}=\cdots \alpha_{n} .
$$

We also put

$$
\beta_{i}=\frac{\alpha_{i}^{\prime} \alpha-n+1}{\sigma-(n+1)(n-2)}=\frac{\alpha_{i}^{\prime} \alpha-n+1}{2(n+1)+\delta} \text { for } 0<i<n
$$

and we note that (3) and (7) imply $\beta_{i} \geq 0$ for $0 \leq i \leq n$ and $\sum_{i=0}^{n} \beta_{i}=1$.

We shall show that the point

$$
z=\sum_{i=0}^{n} \beta_{i} x_{i}
$$

belongs to (4), with

$$
\mu=\frac{\sigma-n(n-2)}{\sigma-(n+1)(n-2)}=\frac{4 n+\delta}{2 n+2+\delta} \leq \frac{2 n}{n+1},
$$

and thus prove the theorem in case (i).

Since all the points $x_{i}$ enter symmetrically in our reasoning, it is sufficient to show that

$$
z \in x_{0}+\mu \alpha_{0} S
$$

the relation (12) will be established by exhibiting a point $y \in x_{0}+\alpha_{0} S$ such that

$$
z-x_{0}=\mu\left(y-x_{0}\right) .
$$

Let $y_{i}=\left(\alpha_{i} x_{0}+\alpha_{0} x_{i}\right) /\left(\alpha_{0}+\alpha_{i}\right)$ for $1 \leq i \leq n$ and

$$
\gamma_{i}=\frac{\left(1+\alpha_{i} \alpha^{\prime}\right)\left(\alpha_{i}^{\prime} \alpha-n+1\right)}{\sigma-n(n-3)}=\frac{\left(1+\alpha_{i} \alpha^{\prime}\right)\left(\alpha_{i}^{\prime} \alpha-n+1\right)}{4 n+\delta}
$$


for $1 \leq i \leq n$. Then $y_{i} \in x_{0}+\alpha_{0} S$ since $\left(x_{0}+\alpha_{0} S\right) \cap\left(x_{i}+\alpha_{i} S\right) \neq \phi$; on the other hand, $\gamma_{i} \geq 0$ because of (3) and (7), and an elementary computation shows that $\sum_{i=1}^{n} \gamma_{i}=1$.

These relations imply that the point $y=\sum_{i=1}^{n} \gamma_{i} y_{i}$ satisfies $y \in x_{0}+\alpha_{0} S$, and equation (13) can be established by a straight-forward computation:

$$
\begin{aligned}
& {[\sigma-n(n-3)]\left(y-x_{0}\right)} \\
& =-[\sigma-n(n-3)] x_{0}+\sum_{i=1}^{n}\left(1+\alpha_{i} \alpha_{0}^{\prime}\right)\left(\alpha_{i}^{\prime} \alpha-n+1\right) \frac{\alpha_{i} x_{0}+\alpha_{0} x_{i}}{\alpha_{i}+\alpha_{0}} \\
& =-[\sigma-n(n-3)] x_{0}+2(n-1) x_{0}+\sum_{i=0}^{n}\left(\alpha \alpha_{i}^{\prime}-n+1\right) x_{i} \\
& {[\sigma-(n+1)(n-2)]\left(\sum_{i=0}^{n} \beta_{i} x_{i}-x_{0}\right)} \\
& =[\sigma-(n+1)(n-2)]\left(z-x_{0}\right) .
\end{aligned}
$$

We turn now to the remaining case (ii). We change, if necessary, the notation in such a way that $0<\alpha_{0} \leq \alpha_{1} \leq \cdots \leq \alpha_{n}$. Then there exists one and only one $k$, with $2 \leq k<n$, such that

$$
(k-1) \alpha_{k} \leq \sum_{i=0}^{k} \alpha_{i}<(k-1) \alpha_{k+1} .
$$

Denoting by $\alpha^{*}, \alpha^{\prime *}, \sigma^{*}, \delta^{*}, \beta^{*}, z^{*}, \mu^{*}$ quantities analogous to those designated by the same letter without asterisk and defined by substituting in (2), (5), (6), (7), (9), (10), and (11) the letter $k$ instead of $n$ and adding asterisks, we may prove as above that

$$
z^{*}=\bigcap_{i=0}^{k}\left(x_{i}+\mu^{*} \alpha_{i} S\right)
$$

with $\mu^{*} \leq \frac{2 k}{k+1} \leq \frac{2 n}{n+1}$.

We shall show that for any $m$ satisfying $k+1 \leq m \leq n$ we have

$$
z^{*} \in x_{m}+\mu_{m} \alpha_{m} S,
$$

where

$$
\mu_{m}=1+\frac{2 \alpha^{*} \alpha_{m}^{\prime}}{\sigma^{*}-(k+1)(k-2)}<1+\frac{2 k-2}{2 k+2+\delta^{*}} \leq \frac{2 k}{k+1}<\frac{2 n}{n+1} .
$$

Indeed, with the notation

$$
\begin{gathered}
y_{i}^{(m)}=\left(\alpha_{m} x_{i}+\alpha_{i} x_{m}\right) /\left(\alpha_{m}+\alpha_{i}\right) \text { for } 0 \leq i \leq k \\
\gamma_{i}^{(m)}=\frac{\left(\alpha_{m}+\alpha_{i}\right)\left(\alpha_{i}^{\prime} \alpha^{*}-k+1\right)}{2 \alpha^{*}+\alpha_{m}\left[\sigma^{*}-(k+1)(k-2)\right]} \text { for } 0 \leq i \leq k
\end{gathered}
$$

it follows as above that 


$$
y_{i}^{(m)} \in x_{m}+\alpha_{m} S, \gamma_{i}^{(m)} \geq 0 \text {, and } \sum_{i=0}^{k} \gamma_{i}^{(m)}=1 \text {. }
$$

Then, defining $y^{(m)}=\sum_{i=0}^{k} \gamma_{i}^{(m)} y_{i}^{(m)}$ we have $y^{(m)} \in x_{m}+\alpha_{m} S$ and

$$
z^{*}-x_{m}=\mu_{m}\left(y^{(m)}-x_{m}\right)
$$

which establishes (14) and thus ends the proof of Theorem 1.

Remarks. 1. From the relation $J_{X} \leq E_{X}$ it follows that Theorem 1 implies Bohnenblust's result $J_{X} \leq \frac{2 n}{n+1}$ for $n$-dimensional $X$. Using appropriate definitions, Leichtweiss [8] showed that the same inequality holds also for $n$-dimensional spaces whose unit cell is not assumed to be symmetric. But it follows easily from these definitions that the unsymmetric case is implied by the symmetric one.

2. Another way to generalize Bohnenblust's result on Jung's constant to spaces with unsymmetric " unit cell" is as follows:

Let $K$ be any convex body in the $n$-dimensional Euclidean space $E^{n}$. $A$ set $M \subset E^{n}$ has $K$-diameter $\leq 1$ if any segment with end-points in $M$ may be covered by a suitable translate of $K$. Any such $M$ may be covered by a translate of $n K$, and the constant $n$ is the best possible.

Indeed, the example of an $n$-dimensional simplex as $K$, and the centrally symmetric simplex as $M$ shows that no number smaller than $n$ is sufficient. (A closer analysis of the following reasoning shows that this is the only case in which $\alpha K$, for a suitable $\alpha<n$, is not sufficient.) In order to prove that any set $M$ of $K$-diameter $\leq 1$ may be covered by a suitable translate of $n K$ we remark that $K$ is contained in a centrally symmetric convex body $K^{*}$ which is contained in a translate of $\frac{1}{2}(n+1) K$. (This result or statements equivalent to it have been proved by many authors; see, e.g., Leichtweiss [8, Lemma 2], Hammer [5], Süss [11], and the references given in these papers.) Then, denoting by $x$ and $y$ suitable points (translations) and using the inequality $J_{K}{ }^{*} \leq \frac{2 n}{n+1}$ we have

$$
M \subset x+\frac{2 n}{n+1} K^{*} \subset y+\frac{2 n}{n+1} \cdot \frac{1}{2}(n+1) K=y+n K,
$$

which proves our assertion.

3. Characterization of the extremal cases. Let $P$ be an $n$-dimensional simplex, and let $S_{P}^{*}=P-P=\{x=y-z ; y, z \in P\}$. Among the $(n-1)$-dimensional faces of $S_{P}^{*}$ there are exactly $2(n+1)$ which are $(n-1)$-dimensional simplices; we denote them by $\pm P_{i}, 0 \leq i \leq n$. Of them, $n+1$ are translates of the $(n-1)$-dimensional faces of $P$, the remaining $n+1$ being centrally symmetric to them. Let $\pm H_{i}$ denote 
the closed half-space bounding $S_{P}^{*}$ and containing $\pm P_{i}$ in its boundary. We define $S_{P}^{* * *}$ as the intersection of all the half-spaces $\pm H_{i}$. Obviously, $S_{P}^{*} \subset S_{P}^{* *}$.

Definition 3. A centrally symmetric convex set $K \subset E^{n}$ is called a Leichtweiss body if and only if for a suitable $n$-dimensional simplex $P$ we have

$$
S_{P}^{*} \subset K \subset S_{P}^{* *}
$$

Now we have the following.

THeOREm 2. The following statements on the n-dimensional Minkowski space $X$ are equivalent:

(a) $J_{X}=2 n /(n+1)$.

(b) $E_{x}=2 n /(n+1)$.

(c) The unit cell $S$ of $X$ is a Leichtweiss body.

Proof. By Theorem 1 and (iii) of the Introduction, (a) implies (b). Let us now assume that (b) is satisfied. Then an inspection of the proof of Theorem 1 shows that there exists a family of cells $\left\{x_{i}+\alpha_{i} S\right\}$ corresponding to case (i) of the proof, and such that equality holds in relations (11) and (8).

Now, since $4 n+\delta /(2 n+2+\delta)<2 n /(n+1)$ for $\delta>0$ (and $n \geq 2$ ) it follows that (b) implies

$$
\alpha_{0}=\alpha_{1}=\cdots=\alpha_{n}
$$

in other words, (a) holds.

On the other hand, (b) implies that $y \in$ Front $\left(x_{0}+\alpha_{0} S\right)$ and that the corresponding relation must hold for the points analogous to $y$ on the other cells. But this implies, because of (15), that $S$ is a Leichtweiss body with $2 P$ equal to the convex hull of the set $\left\{x_{0}, x_{1}, \cdots, x_{n}\right\}$, and therefore (c) follows from (b).

We end the proof of the Theorem 2 by proving that (c) implies (a). Let the simplex $P$ be the convex hull of the points $\left\{x_{i} ; 0 \leq i \leq n\right\}$ and let $S_{P}^{*} \subset S \subset S_{P}^{* *}$. Then $\left\{x_{i}+\frac{1}{2} S ; 0 \leq i \leq n\right\}$ is a family of mutually intersecting cells, since $\left\{x_{i}+\frac{1}{2} S_{P}^{*} ; 0 \leq i \leq n\right\}$ is such a family, but

$$
\bigcap_{i=0}^{n}\left(x_{i}+\frac{1}{2} \mu S\right)=\phi
$$

for any positive $\mu<2 n /(n+1)$ since 


$$
\bigcap_{i=0}^{n}\left(x_{i}+\frac{1}{2} \mu S_{P}^{* *}\right)=\phi
$$

for any such $\mu$.

Thus all the assertions of Theorem 2 are proved.

REMARK. Theorem 2 generalizes the symmetric case of Leichtweiss [8] Satz 2.

4. Expansion constants of Euclidean spaces. In this section we shall strengthen Jung's [7] result by proving the following theorem.

TheOREM 3. $E_{E^{n}}=J_{E^{n}}=\left(\frac{2 n}{n+1}\right)^{1 / 2}$.

Proof. Let $\left\{x_{i}+\alpha_{i} S ; 0 \leq i \leq n\right\}$ be any family of mutually intersecting cells in $E^{n}$ with $\alpha_{i}>0$, and let $\mu$ be the least positive number such that

$$
\bigcap_{i=0}^{n}\left(x_{i}+\mu \alpha_{i} S\right) \neq \phi .
$$

We shall prove Theorem 3 by constructing a family $\left\{y_{i}+S, 0 \leq i \leq n\right\}$ of mutually intersecting cells of equal radius, and such that $\mu$ is least positive number for which

$$
\bigcap_{i=0}^{n}\left(y_{i}+\mu S\right) \neq \phi .
$$

Without loss of generality we may assume that (16) contains only one point $z$ which, moreover, belongs to the boundary of each $x_{i}+\mu \alpha_{i} S$, for $0 \leq i \leq n$. Let $C_{i}$ denote the cone with vertex $z$ generated by $x_{i}+\alpha_{i} S$; then $C_{i} \cap C_{j}$ contains a ray starting at $z$, and all the cones $C_{i}$ are congruent. Now, if $y_{i}=z+(x-z) / \alpha_{i}$, the cell $y_{i}+S$ also generates the cone $C_{i}$ and therefore $(y+S)\left(y_{j}+S\right) \neq \phi$. But obviously $\bigcap_{i=0}^{n}\left(y_{i}+\mu S\right)=\{z\}$, and Theorem 3 is proved.

REMARK. It is well-known that Jung's constant of Hilbert space equals $\sqrt{2}$ (see, e.g., Routledge [10]); it may be shown that its expansion constant also equals $\sqrt{2}$.

5. Concluding remarks. In all the cases discussed above (spaces $X$ with $E_{X}=1$ or $2 n /(n+1)$ and Euclidean spaces) we found that the value of the expansion constant equals the value of Jung's constant. Nevertheless, the two constants are different in general; the simplest example to that effect is perhaps the following: 
Let $Y$ denote the Minkowski plane (with points $(x, y)$ ) whose unit cell is the hexagon with vertices $( \pm 1,0),( \pm \alpha, 1),( \pm \alpha,-1)$ for some $\alpha$ satisfying $0<\alpha<\frac{1}{2}$. We recall the following result (Grünbaum [3]): For any Minkowski plane $X$, if $\rho$ is the minimum of the lengths of sides of equilateral triangles with vertices on the boundary of the unit cell and containing the origin, then $J_{X}=2 / \rho$. It is easily checked that the points $(0 ;-1),(\alpha / 2 ;(2-\alpha) /(2-2 \alpha))$ and $(\alpha / 2 ;-(2-\alpha) /(2-2 \alpha))$ of $Y$, whose mutual distances are $2-\alpha$, determine such a minimal triangle for $H$. Therefore $J_{Y}=2 /(2-\alpha)$. On the other hand, considering the mutually intersecting cells $(1,0)+H,(-1,0)+H$ and $\left(0,1 / \alpha+\frac{1-\alpha}{\alpha} H\right.$, it is immediate that $E_{Y} \geq 1 /\left(1-\alpha+\alpha^{2}\right)$. (It is not difficult to establish that the equality sign holds.) Our assertion then results from the obvious inequality $1 /\left(1-\alpha+\alpha^{2}\right)>2 /(2-\alpha)$.

\section{REFERENCES}

1. N. Aronszajn, and P. Panitchpakdi, Exiension of uniformly continuous transformations and hyperconvex metric spaces, Pacific J. Math., 6 (1956), 405-439.

2. F. Bohnenblust, Convex regions and projections in Minkowski spaces, Ann. of Math., (2) 39 (1938), 301-308.

3. B. Grünbaum, Borsuk's partition conjecture in Minkowski planes, Bull. Research Council of Israel, 7F (1957), 25-30.

4. - Projection constants (in preparation), (Preliminary reports appeared in Technical Notes Nos. 2 and 6, prepared under Contract AF 61 (052)-04, January and May 1958.)

5. P. C. Hammer, The centroid of a convex body, Proc. Amer. Math. Soc., 2 (1951), 522525.

6. O. Hanner, Intersections of translates of convex bodies, Math. Scand., 4. (1956), 65-87,

7. H. W. E. Jung, Ueber die lileinste Kugel, dis eine räumliche Figur einschliesst,

J. Reine. Angew. Math. 123 (1901) 241-257.

8. K. Leichtweiss, Zwei Extremalprobleme der Minkowski-Geometric, Math. Z. 62 (1955), $37-49$.

9. L. Nachbin, A theorem of the Hahn-Banach type for linear transformations, Trans. Amer. Math. Soc., 68 (1950), 28-46.

10. N. A. Routledge, A result in Hilbori spacc, Quart. J. Oxford (2) 3 (1952), 12-18.

11. W. Süss, Ueber cine Affininvarianie von Eibereichen, Arch. Math., 1 (1948) 127-128.

12. B. Szökefalvi-Nagy, Ein Satz ueber Paralleverschiebungen konvexer Körper, Acta Szeged, 15 (1954), 169-177.

THE HeBRew UNIVERSity JeRUSALEM AND

InSTITUTE FOR ADVANCED STUdY

Princeton, NeW JeRseY 


\section{PACIFIC JOURNAL OF MATHEMATICS}

\section{EDITORS}

\section{David Gilbarg}

Stanford University

Stanford, California

\section{R. A. Beaumont}

University of Washington

Seattle 5 , Washington

\author{
A. L. Whiteman
}

University of Southern California Los Angeles 7, California

L. J. Paige

University of California

Los Angeles 24, California

\author{
E. F. BECKENBACH \\ C. E. BURGESS \\ E. HEWITT \\ A. HORN
}

\author{
V. GANAPATHY IYER \\ R. D. JAMES \\ M. S. KNEBELMAN \\ L. NACHBIN
}

ASSOCIATE EDITORS
I. NIVEN

T. G. OSTROM

H. L. ROYDEN

M. M. SCHIFFER
E. G. STRAUS

G. SZEKERES

F. WOLF

K. YOSIDA

\section{SUPPORTING INSTITUTIONS}

\author{
UNIVERSITY OF BRITISH COLUMBIA \\ CALIFORNIA INSTITUTE OF TECHNOLOGY \\ UNIVERSITY OF CALIFORNIA \\ MONTANA STATE UNIVERSITY \\ UNIVERSITY OF NEVADA \\ OREGON STATE COLLEGE \\ UNIVERSITY OF OREGON \\ OSAKA UNIVERSITY \\ UNIVERSITY OF SOUTHERN CALIFORNIA
}

\author{
STANFORD UNIVERSITY \\ UNIVERSITY OF TOKYO \\ UNIVERSITY OF UTAH \\ WASHINGTON STATE COLLEGE \\ UNIVERSITY OF WASHINGTON \\ * * * \\ AMERICAN MATHEMATICAL SOCIETY \\ CALIFORNIA RESEARCH CORPORATION \\ HUGHES AIRCRAFT COMPANY \\ SPACE TECHNOLOGY LABORATORIES
}

Mathematical papers intended for publication in the Pacific Journal of Mathematics should be typewritten (double spaced), and the author should keep a complete copy. Manuscripts may be sent to any one of the four editors. All other communications to the editors should be addressed to the managing editor, L. J. Paige at the University of California, Los Angeles 24, California.

50 reprints per author of each article are furnished free of charge; additional copies may be obtained at cost in multiples of 50 .

The Pacific Journal of Mathematics is published quarterly, in March, June, September, and December. The price per volume (4 numbers) is $\$ 12.00$; single issues, $\$ 3.50$. Back numbers are available. Special price to individual faculty members of supporting institutions and to individual members of the American Mathematical Society: $\$ 4.00$ per volume; single issues, $\$ 1.25$.

Subscriptions, orders for back numbers, and changes of address should be sent to Pacific Journal of Mathematics, 2120 Oxford Street, Berkeley 4, California.

Printed at Kokusai Bunken Insatsusha (International Academic Printing Co., Ltd.), No. 6, 2-chome, Fujimi-cho, Chiyoda-ku, Tokyo, Japan.

PUBLISHED BY PACIFIC JOURNAL OF MATHEMATICS, A NON-PROFIT CORPORATION

The Supporting Institutions listed above contribute to the cost of publication of this Journal, but they are not owners or publishers and have no responsibility for its content or policies. 


\section{Pacific Journal of Mathematics}

\section{Vol. 9, No. $2 \quad$ June, 1959}

Lee William Anderson, On the breadth and co-dimension of a topological lattice

Frank W. Anderson and Robert L. Blair, Characterizations of certain lattices

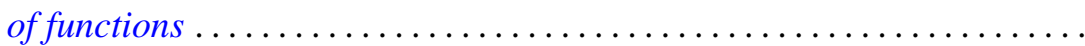

Donald Charles Benson, Extensions of a theorem of Loewner on integral

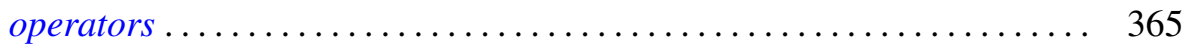

Errett Albert Bishop, A duality theorem for an arbitrary operator ........ 379

Robert McCallum Blumenthal and Ronald Kay Getoor, The asymptotic distribution of the eigenvalues for a class of Markov operators ........

Delmar L. Boyer and Elbert A. Walker, Almost locally pure Abelian

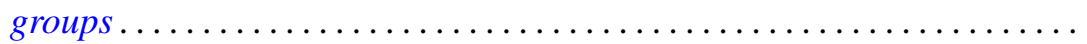

Paul Civin and Bertram Yood, Involutions on Banach algebras ........... Lincoln Kearney Durst, Exceptional real Lehmer sequences .... 415

Eldon Dyer and Allen Lowell Shields, Connectivity of topological

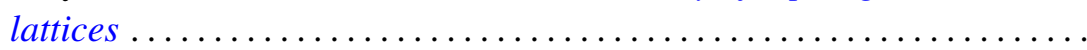

Ronald Kay Getoor, Markov operators and their associated

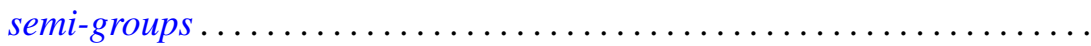

Bernard Greenspan, A bound for the orders of the components of a system of algebraic difference equations

Branko Grünbaum, On some covering and intersection properties in

Minkowski spaces ............................

Bruno Harris, Derivations of Jordan algebras ..............

Henry Berge Helson, Conjugate series in several variables.

Isidore Isaac Hirschman, Jr., A maximal problem in harmonic analysis.

II .

Alfred Horn and Robert Steinberg, Eigenvalues of the unitary part of a matrix

Edith Hirsch Luchins, On strictly semi-simple Banach algebras ...

William D. Munro, Some iterative methods for determining zeros of

functions of a complex variable...

John Rainwater, Spaces whose finest uniformity is metric .

William T. Reid, Variational aspects of generalized convex functions ....

A. Sade, Isomorphisme d'hypergroupoï des isotopes ...... . .

Isadore Manual Singer, The geometric interpretation of a special

connection . . .

Charles Andrew Swanson, Asymptotic perturbation series for characteristic

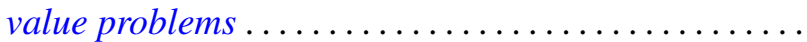

